\title{
Complete Fuzzy n-normed linear space
}

\author{
S. Vijayabalaji ${ }^{a,{ }^{*}}$ and N. Thillaigovindan ${ }^{b,{ }^{*}}$ \\ ${ }^{\text {a }}$ School of Science and Humanities, VIT University, Vellore-632 014, Tamilnadu, India. \\ ${ }^{b}$ Department of Mathematics, Annamalai University, Annamalainagar-608002, Tamilnadu, India. \\ *To whom correspondence should be addressed. E-mail: nandini@rediffmail.com , thillai_n@sify.com \\ Received 12 March 2007 \\ http://dx.doi.org/10.11113/mjfas.v3n1.20
}

\section{ABSTRACT}

This paper introduces the notion of Cauchy sequence, convergent sequence and completeness in fuzzy n-normed linear space.

AMS Mathematics Subject Classification : 46S40, $03 E 72$.

| Fuzzy n-norm | Cauchy sequence | convergent sequence | completeness |

\section{Introduction}

Gahler [4] introduced the theory of n-norm on a linear space. For a systematic development of n-normed linear space one may refer to [5, 6, 8, 9]. In [5], Hendra Gunawan and Mashadi have also discussed the Cauchy sequence and convergent sequence in n-normed linear space. A detailed theory of fuzzy normed linear space can be found in $[1,2,3,7,11]$. In [10], we have extended n-normed linear space to fuzzy n-normed linear space.

Our object in this paper is to introduce the notion of Cauchy sequence and convergent sequence in fuzzy nnormed linear space and to study the completeness of the fuzzy n-normed linear space.

\section{Preliminaries}

Definition 2.1 [5]. Let $\mathrm{n} \in \mathrm{N}$ (natural numbers) and $\mathrm{X}$ be a real linear space of dimension $d \geq \mathrm{n}$. (Here we allow $d$ to be infinite). A real valued function $\|\bullet, \ldots, \bullet\|$ on $\mathrm{X} \times \mathrm{X} \times \ldots \times \mathrm{X}(\mathrm{n}$ times $)=\mathrm{X}^{\mathrm{n}}$ satisfying the following four properties:

(1) $\left\|\mathrm{x}_{1}, \mathrm{x}_{2}, \ldots, \mathrm{x}_{\mathrm{n}}\right\|=0$ if any only if $\mathrm{x}_{1}, \mathrm{x}_{2}, \ldots, \mathrm{x}_{\mathrm{n}}$ are linearly dependent

(2) $\left\|x_{1}, x_{2}, \ldots, x_{n}\right\|$ is invariant under any permutation of $x_{1}, x_{2}, \ldots, x_{n}$ 
(3) $\left\|x_{1}, x_{2}, \ldots, \mathrm{cx}_{n}\right\|=|c|\left\|x_{1}, x_{2}, \ldots, x_{n}\right\|$, for any real $c$

(4) $\left\|\mathrm{x}_{1}, \mathrm{x}_{2}, \ldots, \mathrm{x}_{\mathrm{n}-1}, \mathrm{y}+\mathrm{z}\right\| \leq\left\|\mathrm{x}_{1}, \mathrm{x}_{2}, \ldots, \mathrm{x}_{\mathrm{n}-1}, \mathrm{y}\right\|+\left\|\mathrm{x}_{1}, \mathrm{x}_{2}, \ldots, \mathrm{x}_{\mathrm{n}-1}, \mathrm{z}\right\|$

is called an n-norm on $\mathrm{X}$ and the pair $(\mathrm{X},\|\bullet, \ldots, \bullet\|)$ is called an $\mathrm{n}$ - normed linear space.

Definition 2.2 [5]. A sequence $\left\{x_{n}\right\}$ in an n-normed linear space $(X,\|\bullet, \ldots, \bullet\|)$ is said to converge to an $\mathrm{x} \in \mathrm{X}$ (in the n-norm) whenever $\lim \left\|\mathrm{x}_{1}, \mathrm{x}_{2}, \ldots, \mathrm{x}_{\mathrm{n}-1}, \mathrm{x}_{\mathrm{n}}-\mathrm{x}\right\|=0$.

$$
n \rightarrow \infty
$$

Definition 2.3 [5]. A sequence $\left\{x_{n}\right\}$ in an n-normed linear space $(X,\|\bullet, \ldots, \bullet\|)$ is called a Cauchy sequence if $\lim \left\|x_{1}, x_{2}, \ldots, x_{n-1}, x_{n}-x_{k}\right\|=0$.

$$
\mathrm{n}, \mathrm{k} \rightarrow \infty
$$

Definition 2.4 [5]. An n-normed linear space is said to be complete if every Cauchy sequence in it is convergent.

Definition 2.5 [10]. Let $X$ be a linear space over a real field $F$. A fuzzy subset $N$ of $X^{n} \times R(R$-set of real numbers) is called a fuzzy n-norm on $\mathrm{X}$ if and only if :

(N1) For all $\mathrm{t} \in \mathrm{R}$ with $\mathrm{t} \leq 0, \mathrm{~N}\left(\mathrm{x}_{1}, \mathrm{x}_{2}, \ldots, \mathrm{x}_{\mathrm{n}}, \mathrm{t}\right)=0$.

(N2) For all $\mathrm{t} \in \mathrm{R}$ with $\mathrm{t}>0, \mathrm{~N}\left(\mathrm{x}_{1}, \mathrm{x}_{2}, \ldots, \mathrm{x}_{\mathrm{n}}, \mathrm{t}\right)=1$ if and only if $\mathrm{x}_{1}, \mathrm{x}_{2}, \ldots, \mathrm{x}_{\mathrm{n}}$ are linearly dependent.

(N3) $\quad \mathrm{N}\left(\mathrm{x}_{1}, \mathrm{x}_{2}, \ldots, \mathrm{x}_{\mathrm{n}}, \mathrm{t}\right)$ is invariant under any permutation of $\mathrm{x}_{1}, \mathrm{x}_{2}, \ldots, \mathrm{x}_{\mathrm{n}}$.

(N4) For all $\mathrm{t} \in \mathrm{R}$ with $\mathrm{t}>0$,

$N\left(x_{1}, x_{2}, \ldots, c x_{n}, t\right)=N\left(x_{1}, x_{2}, \ldots, x_{n}, t /|c|\right) \quad$ if $c \neq 0, c \in F$

(N5) For all $\mathrm{s}, \mathrm{t} \in \mathrm{R}$,

$N\left(x_{1}, x_{2}, \ldots, x_{n}+x_{n}^{\prime}, s+t\right) \geq \min \left\{N\left(x_{1}, x_{2}, \ldots, x_{n}, s\right), N\left(x_{1}, x_{2}, \ldots, x_{n}^{\prime}, t\right)\right\}$.

(N6) $\quad \mathrm{N}\left(\mathrm{x}_{1}, \mathrm{x}_{2}, \ldots, \mathrm{x}_{\mathrm{n}}, \mathrm{t}\right)$ is a non-decreasing function of $\mathrm{t} \in \mathrm{R}$ and $\lim \mathrm{N}\left(\mathrm{x}_{1}, \mathrm{x}_{2}, \ldots, \mathrm{x}_{\mathrm{n}}, \mathrm{t}\right)=1$.

$$
\mathrm{t} \rightarrow \infty
$$

Then $(X, N)$ is called a fuzzy n-normed linear space or in short f-n-NLS.

Definition 2.6 [12]. A binary operation $*:[0,1] \times[0,1] \rightarrow[0,1]$ is called a continuous $t$-norm if $*$ satisfies the following conditions:

(1) * is commutative and associative

(2) * is continuous

(3) $a^{*} 1=a$ for all $a \in[0,1]$

(4) $\mathrm{a}^{*} \mathrm{~b} \leq \mathrm{c} * \mathrm{~d}$ whenever $\mathrm{a} \leq \mathrm{c}$ and $\mathrm{b} \leq \mathrm{d}$ and $\mathrm{a}, \mathrm{b}, \mathrm{c}, \mathrm{d} \in[0,1]$. 


\section{Complete fuzzy n-normed linear space}

In this section we first redefine the notion of fuzzy n-normed linear space using t-norm.

Definition 3.1. Let $X$ be a linear space over a real field $F$. A fuzzy subset $N$ of $X^{n} \times[0, \infty)$ is called a fuzzy $\mathrm{n}$-norm on $\mathrm{X}$ if and only if :

$\left(\mathrm{N} 1^{\prime}\right) \mathrm{N}\left(\mathrm{x}_{1}, \mathrm{x}_{2}, \ldots, \mathrm{x}_{\mathrm{n}}, \mathrm{t}\right)>0$.

(N2) $N\left(x_{1}, x_{2}, \ldots, x_{n}, t\right)=1$ if and only if $x_{1}, x_{2}, \ldots, x_{n}$ are linearly dependent.

(N3 $) \mathrm{N}\left(\mathrm{x}_{1}, \mathrm{x}_{2}, \ldots, \mathrm{x}_{\mathrm{n}}, \mathrm{t}\right)$ is invariant under any permutation of $\mathrm{x}_{1}, \mathrm{x}_{2}, \ldots, \mathrm{x}_{\mathrm{n}}$.

$\left(N 4^{\prime}\right) N\left(x_{1}, x_{2}, \ldots, c x_{n}, t\right)=N\left(x_{1}, x_{2}, \ldots, x_{n}, t /|c|\right) \quad$ if $c \neq 0, c \in F($ field $)$.

$\left(N 5^{\prime}\right) \mathrm{N}\left(\mathrm{x}_{1}, \mathrm{x}_{2}, \ldots, \mathrm{x}_{\mathrm{n}}+\mathrm{x}_{\mathrm{n}}^{\prime}, \mathrm{s}+\mathrm{t}\right) \geq \mathrm{N}\left(\mathrm{x}_{1}, \mathrm{x}_{2}, \ldots, \mathrm{x}_{\mathrm{n}}, \mathrm{s}\right) * \mathrm{~N}\left(\mathrm{x}_{1}, \mathrm{x}_{2}, \ldots, \mathrm{x}_{\mathrm{n}}^{\prime}, \mathrm{t}\right)$.

(N6') $\mathrm{N}\left(\mathrm{x}_{1}, \mathrm{x}_{2}, \ldots, \mathrm{x}_{\mathrm{n}}, \mathrm{t}\right)$ is left continuous and non-decreasing function such that $\lim N\left(x_{1}, x_{2}, \ldots, x_{n}, t\right)=1$.

$t \rightarrow \infty$

Then $(\mathrm{X}, \mathrm{N})$ is called a fuzzy n-normed linear space or in short f-n-NLS.

To strengthen the above definition, we present the following example.

Example 3. 2. Let $(X,\|\bullet, \ldots, \bullet\|)$ be an n-normed linear space.

Define $\mathrm{a} * \mathrm{~b}=\min \{\mathrm{a}, \mathrm{b}\}$ and $\mathrm{N}\left(\mathrm{x}_{1}, \mathrm{x}_{2}, \ldots, \mathrm{x}_{\mathrm{n}}, \mathrm{t}\right)=\mathrm{t} /\left(\mathrm{t}+\left\|\mathrm{x}_{1}, \mathrm{x}_{2}, \ldots, \mathrm{x}_{\mathrm{n}}\right\|\right)$.

Then $(X, N)$ is a $\mathrm{f}-\mathrm{n}-\mathrm{NLS}$.

\section{Proof.}

(N1') Clearly $\mathrm{N}\left(\mathrm{x}_{1}, \mathrm{x}_{2}, \ldots, \mathrm{x}_{\mathrm{n}}, \mathrm{t}\right)>0$.

$\left(\mathrm{N} 2^{\prime}\right) \mathrm{N}\left(\mathrm{x}_{1}, \mathrm{x}_{2}, \ldots, \mathrm{x}_{\mathrm{n}}, \mathrm{t}\right)=1$

$\Leftrightarrow \quad \mathrm{t} /\left(\mathrm{t}+\left\|\mathrm{x}_{1}, \mathrm{x}_{2}, \ldots, \mathrm{x}_{\mathrm{n}}\right\|\right)=1$

$\Leftrightarrow \quad\left\|x_{1}, x_{2}, \ldots, x_{n}\right\|=0$

$\Leftrightarrow \quad \mathrm{x}_{1}, \mathrm{x}_{2}, \ldots, \mathrm{x}_{\mathrm{n}}$ are linearly dependent.

(N3') $\mathrm{N}\left(\mathrm{x}_{1}, \mathrm{x}_{2}, \ldots, \mathrm{x}_{\mathrm{n}}, \mathrm{t}\right)$

$=\mathrm{t} /\left(\mathrm{t}+\left\|\mathrm{x}_{1}, \mathrm{x}_{2}, \ldots, \mathrm{x}_{\mathrm{n}}\right\|\right)$

$=\mathrm{t} /\left(\mathrm{t}+\left\|\mathrm{x}_{1}, \mathrm{x}_{2}, \ldots, \mathrm{x}_{\mathrm{n}}, \mathrm{x}_{\mathrm{n}-1}\right\|\right)$

$=\mathrm{N}\left(\mathrm{x}_{1}, \mathrm{x}_{2}, \ldots, \mathrm{x}_{\mathrm{n}}, \mathrm{x}_{\mathrm{n}-1}, \mathrm{t}\right)$. 
It follows similarly for the rest.

$\left(\mathrm{N} 4^{\prime}\right) \mathrm{N}\left(\mathrm{x}_{1}, \mathrm{x}_{2}, \ldots, \mathrm{x}_{\mathrm{n}}, \mathrm{t} /|\mathrm{c}|\right)$

$=(\mathrm{t} /|\mathrm{c}|) /\left(\mathrm{t} /|\mathrm{c}|+\left\|\mathrm{x}_{1}, \mathrm{x}_{2}, \ldots, \mathrm{x}_{\mathrm{n}}\right\|\right)$

$=\mathrm{t} /\left(\mathrm{t}+|\mathrm{c}|\left\|\mathrm{x}_{1}, \mathrm{x}_{2}, \ldots, \mathrm{x}_{\mathrm{n}}\right\|\right)$

$=\mathrm{t} /\left(\mathrm{t}+\left\|\mathrm{x}_{1}, \mathrm{x}_{2}, \ldots, \mathrm{cx}_{\mathrm{n}}\right\|\right)$

$=\mathrm{N}\left(\mathrm{x}_{1}, \mathrm{x}_{2}, \ldots, \mathrm{cx}_{\mathrm{n}}, \mathrm{t}\right)$.

(N5 ' $)$ Without loss of generality assume that $N\left(x_{1}, x_{2}, \ldots, x_{n}^{\prime}, t\right) \leq N\left(x_{1}, x_{2}, \ldots, x_{n}, s\right)$. Then

$\mathrm{t} /\left(\mathrm{t}+\left\|\mathrm{x}_{1}, \mathrm{x}_{2}, \ldots, \mathrm{x}_{\mathrm{n}}^{\prime}\right\|\right) \leq \mathrm{s} /\left(\mathrm{s}+\left\|\mathrm{x}_{1}, \mathrm{x}_{2}, \ldots, \mathrm{x}_{\mathrm{n}}\right\|\right)$

$\Rightarrow \mathrm{t} \quad\left(\mathrm{s}+\left\|\mathrm{x}_{1}, \mathrm{x}_{2}, \ldots, \mathrm{x}_{\mathrm{n}}\right\|\right) \leq \mathrm{s}\left(\mathrm{t}+\left\|\mathrm{x}_{1}, \mathrm{x}_{2}, \ldots, \mathrm{x}_{\mathrm{n}}^{\prime}\right\|\right)$

$\Rightarrow \mathrm{t}\left\|\mathrm{x}_{1}, \mathrm{x}_{2}, \ldots, \mathrm{x}_{\mathrm{n}}\right\| \leq \mathrm{s}\left\|\mathrm{x}_{1}, \mathrm{x}_{2}, \ldots, \mathrm{x}_{\mathrm{n}}^{\prime}\right\|$

$\Rightarrow\left\|\mathrm{x}_{1}, \mathrm{x}_{2}, \ldots, \mathrm{x}_{\mathrm{n}}\right\| \leq(\mathrm{s} / \mathrm{t})\left\|\mathrm{x}_{1}, \mathrm{x}_{2}, \ldots, \mathrm{x}_{\mathrm{n}}^{\prime}\right\|$.

Therefore,

$$
\begin{aligned}
& \left\|\mathrm{x}_{1}, \mathrm{x}_{2}, \ldots, \mathrm{x}_{\mathrm{n}}\right\|+\left\|\mathrm{x}_{1}, \mathrm{x}_{2}, \ldots, \mathrm{x}_{\mathrm{n}}^{\prime}\right\| \\
& \leq(\mathrm{s} / \mathrm{t})\left\|\mathrm{x}_{1}, \mathrm{x}_{2}, \ldots, \mathrm{x}_{\mathrm{n}}^{\prime}\right\|+\left\|\mathrm{x}_{1}, \mathrm{x}_{2}, \ldots, \mathrm{x}_{\mathrm{n}}^{\prime}\right\| \\
& \leq(\mathrm{s} / \mathrm{t}+1)\left\|\mathrm{x}_{1}, \mathrm{x}_{2}, \ldots, \mathrm{x}_{\mathrm{n}}^{\prime}\right\| \\
& =((\mathrm{s}+\mathrm{t}) / \mathrm{t})\left\|\mathrm{x}_{1}, \mathrm{x}_{2}, \ldots, \mathrm{x}_{\mathrm{n}}^{\prime}\right\|
\end{aligned}
$$

But,

$$
\begin{aligned}
& \left\|x_{1}, x_{2}, \ldots, x_{n}+x_{n}^{\prime}\right\| \\
& \leq\left\|x_{1}, x_{2}, \ldots, x_{n}\right\|+\left\|x_{1}, x_{2}, \ldots, x^{\prime}{ }_{n}\right\| \\
& \leq((s+t) / t)\left\|x_{1}, x_{2}, \ldots, x_{n}^{\prime}\right\| . \\
& \Rightarrow\left(\left\|x_{1}, x_{2}, \ldots, x_{n}+x_{n}^{\prime}\right\|\right) /(s+t) \leq\left(\left\|x_{1}, x_{2}, \ldots, x_{n}^{\prime}\right\|\right) / t \\
& \Rightarrow 1+\left(\left\|x_{1}, x_{2}, \ldots, x_{n}+x_{n}^{\prime}\right\|\right) /(s+t) \leq 1+\left(\left\|x_{1}, x_{2}, \ldots, x_{n}^{\prime}\right\|\right) / t \\
& \Rightarrow\left((s+t)+\left\|x_{1}, x_{2}, \ldots, x_{n}+x_{n}^{\prime}\right\|\right) /(s+t) \leq\left(t+\left\|x_{1}, x_{2}, \ldots, x_{n}^{\prime}\right\|\right. \\
& \Rightarrow(s+t) /\left(\left\|x_{1}, x_{2}, \ldots, x_{n}+x_{n}^{\prime}\right\|+s+t\right) \geq t /\left(t+\left\|x_{1}, x_{2}, \ldots, x_{n}^{\prime}\right\|\right) \\
& \Rightarrow N\left(x_{1}, x_{2}, \ldots, x_{n}+x_{n}^{\prime}, s+t\right) \geq \min \left\{N\left(x_{1}, x_{2}, \ldots, x_{n}, s\right), N\left(x_{1}, x_{2}, \ldots, x_{n}^{\prime}, t\right)\right\} .
\end{aligned}
$$

(N6 ' $)$ Clearly $\mathrm{N}\left(\mathrm{x}_{1}, \mathrm{x}_{2}, \ldots, \mathrm{x}_{\mathrm{n}}, \mathrm{t}\right)$ is a left continuous function.

Suppose that $\mathrm{t}_{2}>\mathrm{t}_{1}>0$ with $\mathrm{t}_{1}, \mathrm{t}_{2} \in[0, \infty)$ then,

$$
\begin{aligned}
& \mathrm{t}_{2} /\left(\mathrm{t}_{2}+\left\|\mathrm{x}_{1}, \mathrm{x}_{2}, \ldots, \mathrm{x}_{\mathrm{n}}\right\|\right)-/ \mathrm{t}_{1}\left(\mathrm{t}_{1}+\left\|\mathrm{x}_{1}, \mathrm{x}_{2}, \ldots, \mathrm{x}_{\mathrm{n}}\right\|\right) \\
& =\left\|\mathrm{x}_{1}, \mathrm{x}_{2}, \ldots, \mathrm{x}_{\mathrm{n}}\right\|\left(\mathrm{t}_{2}-\mathrm{t}_{1}\right) /\left(\left(\mathrm{t}_{2}+\left\|\mathrm{x}_{1}, \mathrm{x}_{2}, \ldots, \mathrm{x}_{\mathrm{n}}\right\|\right)\left(\mathrm{t}_{1}+\left\|\mathrm{x}_{1}, \mathrm{x}_{2}, \ldots, \mathrm{x}_{\mathrm{n}}\right\|\right)\right) \geq 0, \\
& \text { for all }\left(\mathrm{x}_{1}, \mathrm{x}_{2}, \ldots, \mathrm{x}_{\mathrm{n}}\right) \in \mathrm{X}^{\mathrm{n}} \\
& \Rightarrow \mathrm{t}_{2} /\left(\mathrm{t}_{2}+\left\|\mathrm{x}_{1}, \mathrm{x}_{2}, \ldots, \mathrm{x}_{\mathrm{n}}\right\|\right) \geq / \mathrm{t}_{1} /\left(\mathrm{t}_{1}+\left\|\mathrm{x}_{1}, \mathrm{x}_{2}, \ldots, \mathrm{x}_{\mathrm{n}}\right\|\right)
\end{aligned}
$$


$\Rightarrow \mathrm{N}\left(\mathrm{x}_{1}, \mathrm{x}_{2}, \ldots, \mathrm{x}_{\mathrm{n}}, \mathrm{t}_{2}\right) \geq \mathrm{N}\left(\mathrm{x}_{1}, \mathrm{x}_{2}, \ldots, \mathrm{x}_{\mathrm{n}}, \mathrm{t}_{1}\right)$.

Thus $\mathrm{N}\left(\mathrm{x}_{1}, \mathrm{x}_{2}, \ldots, \mathrm{x}_{\mathrm{n}}, \mathrm{t}\right)$ is a non-decreasing function of $\mathrm{t} \in[0, \infty)$.

Also,

$$
\begin{aligned}
& \lim _{t \rightarrow \infty} N\left(x_{1}, x_{2}, \ldots, x_{n}, t\right) \\
& =\lim _{t \rightarrow \infty} t /\left(t+\left\|x_{1}, x_{2}, \ldots, x_{n}\right\|\right) \\
& =\lim _{t \rightarrow \infty} t / t\left(1+1 / t\left\|x_{1}, x_{2}, \ldots, x_{n}\right\|\right) \\
& =1 .
\end{aligned}
$$

Thus, $\quad(X, N)$ is a $f-n-N L S$.

Definition 3.3. A sequence $\left\{x_{n}\right\}$ in a $f-n-N L S(X, N)$ is said to converge to $x$ if given $r>0, t>0,0<r<1$, there exists an integer $n_{0} \in N$ such that $N\left(x_{1}, x_{2}, \ldots, x_{n-1}, x_{n}-x, t\right)>1-r$ for all $n \geq n_{0}$.

Theorem 3.4. In a $f-n-N L S(X, N)$ a sequence $\left\{x_{n}\right\}$ converges to $x$ if and only if $\mathrm{N}\left(\mathrm{x}_{1}, \mathrm{x}_{2}, \ldots, \mathrm{x}_{\mathrm{n}-1}, \mathrm{x}_{\mathrm{n}}-\mathrm{x}, \mathrm{t}\right) \rightarrow 1$ as $\mathrm{n} \rightarrow \infty$.

\section{Proof.}

Fix $\mathrm{t}>0$. Suppose $\left\{\mathrm{x}_{\mathrm{n}}\right\}$ converges to $\mathrm{x}$.

Then for a given $r, 0<r<1$, there exists an integer $n_{0} \in N$ such that $N\left(x_{1}, x_{2}, \ldots, x_{n-1}, x_{n}-x, t\right)>1-r$.

Thus $1-\mathrm{N}\left(\mathrm{x}_{1}, \mathrm{x}_{2}, \ldots, \mathrm{x}_{\mathrm{n}-1}, \mathrm{x}_{\mathrm{n}}-\mathrm{x}, \mathrm{t}\right)<\mathrm{r}$ and hence $\mathrm{N}\left(\mathrm{x}_{1}, \mathrm{x}_{2}, \ldots, \mathrm{x}_{\mathrm{n}-1}, \mathrm{x}_{\mathrm{n}}-\mathrm{x}, \mathrm{t}\right) \rightarrow 1$ as $\mathrm{n} \rightarrow \infty$.

Conversely, if for each $\mathrm{t}>0, \mathrm{~N}\left(\mathrm{x}_{1}, \mathrm{x}_{2}, \ldots, \mathrm{x}_{\mathrm{n}-1}, \mathrm{x}_{\mathrm{n}}-\mathrm{x}, \mathrm{t}\right) \rightarrow 1$ as $\mathrm{n} \rightarrow \infty$, then for every $\mathrm{r}, 0<\mathrm{r}<1$, there exists an integer $\mathrm{n}_{0}$ such that $1-\mathrm{N}\left(\mathrm{x}_{1}, \mathrm{x}_{2}, \ldots, \mathrm{x}_{\mathrm{n}-1}, \mathrm{x}_{\mathrm{n}}-\mathrm{x}, \mathrm{t}\right)<\mathrm{r}$ for all $n \geq n_{0}$. Thus $N\left(x_{1}, x_{2}, \ldots, x_{n-1}, x_{n}-x, t\right)>1-r$ for all $n \geq n_{0}$.

Hence $\left\{x_{n}\right\}$ converges to $x$ in $(X, N)$.

Definition 3.5. A sequence $\left\{x_{n}\right\}$ in a f-n-NLS (X,N) is said to be Cauchy sequence if given $\varepsilon>0$ with $0<\varepsilon<$ $1, t>0$, there exists an integer $n_{0} \in N$ such that $N\left(x_{1}, x_{2}, \ldots, x_{n-1}, x_{n}-x_{k}, t\right)>1-\varepsilon$ for all $n, k \geq n_{0}$.

Theorem 3.6. In a f-n-NLS $(X, N)$ every convergent sequence is a Cauchy sequence.

Proof. Let $\left\{\mathrm{x}_{\mathrm{n}}\right\}$ be a convergent sequence in $(\mathrm{X}, \mathrm{N})$. Suppose $\left\{\mathrm{x}_{\mathrm{n}}\right\}$ converges to $\mathrm{x}$.

Let $t>0$ and $\varepsilon \in(0,1)$. Choose $r \in(0,1)$ such that $(1-r) *(1-r)>1-\varepsilon$.

Since $\left\{\mathrm{x}_{\mathrm{n}}\right\}$ converges to $\mathrm{x}$, we have an integer $\mathrm{n}_{0}$ such that $\mathrm{N}\left(\mathrm{x}_{1}, \mathrm{x}_{2}, \ldots, \mathrm{x}_{\mathrm{n}-1}, \mathrm{x}_{\mathrm{n}}-\mathrm{x}, \mathrm{t} / 2\right)>1-\mathrm{r}$.

Now, $N\left(x_{1}, x_{2}, \ldots, x_{n-1}, x_{n}-x_{k}, t\right)$

$=N\left(x_{1}, x_{2}, \ldots, x_{n-1}, x_{n}-x+x-x_{k}, t\right)$

$=\mathrm{N}\left(\mathrm{x}_{1}, \mathrm{x}_{2}, \ldots, \mathrm{x}_{\mathrm{n}-1}, \mathrm{x}_{\mathrm{n}}-\mathrm{x}, \mathrm{t} / 2\right) * \mathrm{~N}\left(\mathrm{x}_{1}, \mathrm{x}_{2}, \ldots, \mathrm{x}_{\mathrm{n}-1}, \mathrm{x}-\mathrm{x}_{\mathrm{k}}, \mathrm{t} / 2\right)$ 
$\geq(1-r) *(1-r)$ for all $\mathrm{n}, \mathrm{k} \geq \mathrm{n}_{0}$

$>1-\varepsilon$ for all $n, k \geq n_{0}$.

Therefore $\left\{x_{n}\right\}$ is a Cauchy sequence in $(X, N)$.

Definition 3.7. A f-n-NLS is said to be complete if every Cauchy sequence in it is convergent.

The following example shows that there may exist Cauchy sequence in a f-n-NLS which is not convergent.

Example 3. 8. Let $(X,\|\bullet, \ldots, \bullet\|)$ be an n-normed linear space and define $a * b=\min \{a, b\}$ for all $a, b \in[0,1]$ and $\mathrm{N}\left(\mathrm{x}_{1}, \mathrm{x}_{2}, \ldots, \mathrm{x}_{\mathrm{n}}, \mathrm{t}\right)=\mathrm{t} /\left(\mathrm{t}+\left\|\mathrm{x}_{1}, \mathrm{x}_{2}, \ldots, \mathrm{x}_{\mathrm{n}}\right\|\right)$. Then $(\mathrm{X}, \mathrm{N})$ is shown to be a $\mathrm{f}-\mathrm{n}-\mathrm{NLS}$.

Let $\left\{x_{n}\right\}$ be a sequence in $\mathrm{f}-\mathrm{n}-\mathrm{NLS}$, then

(a) $\left\{\mathrm{x}_{\mathrm{n}}\right\}$ is a Cauchy sequence in $(\mathrm{X},\|\bullet, \ldots, \bullet\|)$ if and only if $\left\{\mathrm{x}_{\mathrm{n}}\right\}$ is a Cauchy sequence in $(\mathrm{X}, \mathrm{N})$.

(b) $\left\{x_{n}\right\}$ is a convergent sequence in $(X,\|\bullet, \ldots, \bullet\|)$ if and only if $\left\{x_{n}\right\}$ is a convergent sequence in $(\mathrm{X}, \mathrm{N})$.

\section{Proof.}

(a) $\left\{\mathrm{X}_{\mathrm{n}}\right\}$ is a Cauchy sequence in $(\mathrm{X},\|\bullet, \ldots, \bullet\|)$

$$
\begin{aligned}
& \Leftrightarrow \lim _{n, k \rightarrow \infty}\left\|x_{1}, x_{2}, \ldots, x_{n-1}, x_{n}-x_{k}\right\|=0 . \\
& \Leftrightarrow \quad \lim _{n, k \rightarrow \infty} N\left(x_{1}, x_{2}, \ldots, x_{n-1}, x_{n}-x_{k}, t\right) \\
& =\quad \lim _{n, k \rightarrow \infty} t /\left(t+\left\|x_{1}, x_{2}, \ldots, x_{n-1}, x_{n}-x_{k}\right\|\right)=1 . \\
& \Leftrightarrow \quad N\left(x_{1}, x_{2}, \ldots, x_{n-1}, x_{n}-x_{k}, t\right) \rightarrow 1 \quad \text { as } n \rightarrow \infty . \\
& \Leftrightarrow \quad N\left(x_{1}, x_{2}, \ldots, x_{n-1}, x_{n}-x_{k}, t\right)>1-r, \text { for all } n, k \geq n_{0} . \\
& \Leftrightarrow \quad\left\{x_{n}\right\} \text { is a Cauchy sequence in }(X, N) .
\end{aligned}
$$

(b) $\left\{\mathrm{X}_{\mathrm{n}}\right\}$ is a convergent sequence in $(\mathrm{X},\|\bullet, \ldots, \bullet\|)$

$$
\begin{aligned}
& \Leftrightarrow \lim _{\mathrm{n} \rightarrow \infty}\left\|\mathrm{x}_{1}, \mathrm{x}_{2}, \ldots, \mathrm{x}_{\mathrm{n}-1}, \mathrm{x}_{\mathrm{n}}-\mathrm{x}\right\|=0 . \\
& \Leftrightarrow \lim _{\mathrm{n} \rightarrow \infty} \mathrm{N}\left(\mathrm{x}_{1}, \mathrm{x}_{2}, \ldots, \mathrm{x}_{\mathrm{n}-1}, \mathrm{x}_{\mathrm{n}}-\mathrm{x}_{\mathrm{k}}, \mathrm{t}\right) \\
& =\lim _{\mathrm{n} \rightarrow \infty} \mathrm{t} /\left(\mathrm{t}+\left\|\mathrm{x}_{1}, \mathrm{x}_{2}, \ldots, \mathrm{x}_{\mathrm{n}-1}, \mathrm{x}_{\mathrm{n}}-\mathrm{x}_{\mathrm{k}}\right\|\right)=1 .
\end{aligned}
$$




$$
\begin{aligned}
& \Leftrightarrow N\left(x_{1}, x_{2}, \ldots, x_{n-1}, x_{n}-x, t\right) \rightarrow 1 \text { as } n \rightarrow \infty . \\
& \Leftrightarrow N\left(x_{1}, x_{2}, \ldots, x_{n-1}, x_{n}-x, t\right)>1-r \text {, for all } n \geq n_{0} . \\
& \Leftrightarrow\left\{x_{n}\right\} \text { is a convergent sequence in }(X, N) .
\end{aligned}
$$

Thus if there exists an n-normed linear space $(X,\|\bullet, \ldots, \bullet\|)$ which is not complete, then the fuzzy n-norm induced by such a crisp n-norm $\|\bullet, \ldots, \bullet\|$ on an incomplete $n$-normed linear space $\mathrm{X}$ is an incomplete fuzzy $\mathrm{n}$ normed linear space.

Theorem 3.9. $\quad$ A f-n-NLS (X, N ) in which every Cauchy sequence has a convergent subsequence is complete.

\section{Proof.}

Let $\left\{x_{n}\right\}$ be a Cauchy sequence in $(X, N)$ and $\left\{x_{n_{k}}\right\}$ be a subsequence of $\left\{x_{n}\right\}$ that converges to $x$. We prove that $\left\{\mathrm{x}_{\mathrm{n}}\right\}$ converges to $\mathrm{x}$. Let $\mathrm{t}>0$ and $\varepsilon \in(0,1)$. Choose $\mathrm{r} \in(0,1)$ such that $(1-\mathrm{r}) *(1-\mathrm{r})>1-\varepsilon$. Since $\left\{\mathrm{x}_{\mathrm{n}}\right\}$ is a Cauchy sequence, there exists an integer $\mathrm{n}_{0} \in \mathrm{N}$ such that $\mathrm{N}\left(\mathrm{x}_{1}, \mathrm{x}_{2}, \ldots, \mathrm{x}_{\mathrm{n}-1}, \mathrm{x}_{\mathrm{n}}-\mathrm{x}_{\mathrm{k}}, \mathrm{t} / 2\right)>1-\mathrm{r}$ for all $\mathrm{n}, \mathrm{k} \geq \mathrm{n}_{0}$. Since $\left\{\mathrm{x}_{\mathrm{n}_{k}}\right\}$ converges to $\mathrm{x}$, there is a positive integer $\mathrm{i}_{\mathrm{k}}>\mathrm{n}_{0}$ such that $\mathrm{N}\left(\mathrm{x}_{1}, \mathrm{x}_{2}, \ldots, \mathrm{x}_{\mathrm{n}-1}, \mathrm{x}_{\mathrm{i}_{k}}-\mathrm{x}, \mathrm{t} / 2\right)>1-\mathrm{r}$.

Now,

$$
\begin{aligned}
& \mathrm{N}\left(\mathrm{x}_{1}, \mathrm{x}_{2}, \ldots, \mathrm{x}_{\mathrm{n}-1}, \mathrm{x}_{\mathrm{n}}-\mathrm{x}, \mathrm{t} / 2\right) \\
& =\mathrm{N}\left(\mathrm{x}_{1}, \mathrm{x}_{2}, \ldots, \mathrm{x}_{\mathrm{n}-1}, \mathrm{x}_{\mathrm{n}}-\mathrm{x}_{\mathrm{i}_{k}}+\mathrm{x}_{\mathrm{i}_{k}}-\mathrm{x}, \mathrm{t} / 2+\mathrm{t} / 2\right) \\
& \geq \mathrm{N}\left(\mathrm{x}_{1}, \mathrm{x}_{2}, \ldots, \mathrm{x}_{\mathrm{n}-1}, \mathrm{x}_{\mathrm{n}}-\mathrm{x}_{\mathrm{i}_{k}}, \mathrm{t} / 2\right) * \mathrm{~N}\left(\mathrm{x}_{1}, \mathrm{x}_{2}, \ldots, \mathrm{x}_{\mathrm{n}-1}, \mathrm{x}_{\mathrm{i}_{k}}-\mathrm{x}, \mathrm{t} / 2\right) \\
& >(1-\mathrm{r}) *(1-\mathrm{r}) \\
& >1-\varepsilon .
\end{aligned}
$$

Therefore $\left\{\mathrm{x}_{\mathrm{n}}\right\}$ converges to $\mathrm{x}$ in $(\mathrm{X}, \mathrm{N})$ and hence it is complete.

\section{References}

[1] T.Bag and S.K.Samanta, Finite dimensional fuzzy normed linear spaces, The Journal of Fuzzy Mathematics, 11 ( 2003), No.3, 687-705.

[2] S.C.Chang and J.N.Mordesen, Fuzzy linear operators and fuzzy normed linear spaces, Bull.Cal.Math.Soc., 86 (1994), 429-436.

[3] C.Felbin, Finite dimensional fuzzy normed linear spaces II, Journal of Analysis, (1999), 117-131.

[4] S.Gähler, Unter Suchungen Über Veralla gemeinerte m-metrische Räume I, Math.Nachr., 40 (1969), 165-189.

[5] Hendra Gunawan and M.Mashadi, on n-normed spaces, International J. Math. \& Math. Sci., 27 (2001), No.10, 631-639.

[6] S.S.Kim and Y.J.Cho, Strict convexity in linear n-normed spaces, Demonstratio Math., 29 (1996), No.4, 739-744.

[7] S.V.Krishna and K.K.M.Sarma, Separation of fuzzy normed linear spaces, Fuzzy Sets and Systems, 63 (1994), 207-217. 
[8] R.Malceski, Strong n-convex n-normed spaces,Mat.Bilten, 21 (1997), 81-102.

[9] A.Misiak, n-inner product spaces, Math.Nachr., 140 (1989), 299-319.

[10] AL.Narayanan and S.Vijayabalaji, Fuzzy n-normed linear space, International J. Math. \& Math. Sci., 2005, No.24, 3963-3977.

[11] G.S.Rhie, B.M.Choi and S.K.Dong, On the completeness of fuzzy normed linear spaces, Math. Japonica, 45 (1997), No.1, 33-37.

[12] B.Schweizer and A.Sklar, Statistical metric spaces, Pacific J.Math., 10 (1960) 314-334. 